\title{
1 Long read metagenomics, the next step?
}

2 Jose M. Haro-Moreno ${ }^{\mathrm{a}, \ddagger}$, Mario López-Pérez ${ }^{\mathrm{a}, \ddagger}$ and Francisco Rodríguez-Valeraa,b,\#.

aEvolutionary Genomics Group, División de Microbiología, Universidad Miguel

5 Hernández, Apartado 18, San Juan 03550, Alicante, Spain. ${ }^{\mathrm{b}}$ Research Center for

6 Molecular Mechanisms of Aging and Age-related Diseases, Moscow Institute of

7 Physics and Technology, Dolgoprudny, 141701 Russia. 需ese authors contributed

8 equally to this work.

$10 \quad$ \#Corresponding author: frvalera@umh.es

11 Evolutionary Genomics Group, División de Microbiología, Universidad Miguel

12 Hernández, Apartado 18, San Juan de Alicante, 03550 Alicante, Spain.

14 Running title: long read metagenomics

16 Keywords: Metagenome, metagenome-assembled genome, long-reads, Nanopore,

17 PacBio, rhodopsin, polyketide synthase, CRISPR

\section{ABSTRACT}

\section{Background}

21 Third-generation sequencing has penetrated little in metagenomics due to the high error rate and dependence for assembly on short-read designed bioinformatics. However, 2nd generation sequencing metagenomics (mostly Illumina) suffers from

24 limitations, particularly in allowing assembly of microbes with high microdiversity or 25 retrieving the flexible (adaptive) compartment of prokaryotic genomes. 


\section{Results}

27 Here we have used different 3rd generation techniques to study the metagenome of a well-known marine sample from the mixed epipelagic water column of the winter Mediterranean. We have compared Oxford Nanopore and PacBio last generation

30 technologies with the classical approach using Illumina short reads followed by assembly. PacBio Sequel II CCS appears particularly suitable for cellular metagenomics due to its low error rate. Long reads allow efficient direct retrieval of complete genes (473M/Tb) and operons before assembly, facilitating annotation and compensates the limitations of short reads or short-read assemblies. MetaSPAdes was the most appropriate assembly program when used in combination with short reads. The assemblies of the long reads allow also the reconstruction of much more complete metagenome-assembled genomes, even from microbes with high microdiversity. The flexible genome of reconstructed MAGs is much more complete and allows rescuing more adaptive genes.

\section{Conclusions}

41 For most applications of metagenomics, from community structure analysis to ecosystem functioning, long-reads should be applied whenever possible. Particularly for in-silico screening of biotechnologically useful genes, or population genomics, long-read metagenomics appears presently as a very fruitful approach and can be used from raw reads, before a computing-demanding (and potentially artefactual) assembly step. 


\section{BACKGROUND}

Metagenomics is still among the most powerful tools of exploratory Microbiology. Its application to several environments has allowed enlarging enormously what we know about the real (and largely unexpected) diversity of prokaryotic cells [1,2]. In actuality, these advances were largely possible by the advent of Illumina, high-throughput lowerror short-read (SR) sequencing that has allowed enormous datasets that can be used for assembly of mock genomes called metagenome-assembled genomes (MAGs) $[3,4]$. Often supported by, typically incomplete, and expensive to generate, but largely reliable, single-cell amplified genomes (SAGs) [5]. MAGs have allowed rewriting most of what we knew about microbes during the last 10 years [6]. However, assembly driven metagenomics has weaknesses, i) low recovery of high microdiversity microbes [7], ii) low recovery of the flexible genome [8] and iii) uncertainty due to potential chimera generation [9].

Long-read (LR) sequencing (i.e. Oxford Nanopore technologies -Nanopore, and Pacific Biosciences - PacBio) $[10,11]$ solves major problems for genome assembly by covering large genomic tracks including the short to medium size repeats that mystify Illumina assembly algorithms [12-14]. Thus, it allows extremely fast and accurate closing of viral, prokaryotic, or even eukaryotic genomes [15-18]. However, these techniques are in general much more prone to error than Illumina what complicates their application for metagenomics. Therefore, high coverage of a homogeneous sequence is a must to get a reasonably reliable sequence [19]. However, the recent development of PacBio Sequel II chemistry allows decreasing enormously the error rate [20]. LR sequencing has the potential of fixing the problems of SR assembly and it also offers a good complementarity to SAGs since it is not biased by an amplification step and is simpler and much cheaper. LR metagenomes can be annotated directly 
from the sequence output avoiding erroneous protein translation and call. This would allow a good metabolic reconstruction of the environment with a high-accuracy prediction of biochemical activities. The core genome, the part best reconstructed in MAGs, is often the least interesting for ecological/biotechnological applications but could be reconstructed and exploited using LR. High reliable taxonomic affiliation by consensus similarity of multiple genes to a reference genome allows better inference of the origin of individual genes. Taxonomy markers such as ribosomal RNA operons can be retrieved complete allowing a reliable community structure determination [21]. To assess the resolving power of the two most advanced LR sequencing available (Oxford Nanopore and PacBio Sequel II) and compare it with Illumina we have selected a real metagenome rather than artificial genome mixtures [22-24] or low diversity environments $[25,26]$. We still do not know the real extent of the diversity of a real-life complex community to be able to mimic it with mixtures of known genomes. Besides, this kind of test have already been done and provided satisfactory results [22-24]. The open ocean is one of the oldest and most important communities for the global ecology of the planet and has been extensively studied by several methods, including metagenomics, for decades [27-30]. We took a sample from offshore Mediterranean waters in winter, when the water column is mixed, and it is likely that any depth sampled would provide a richer representation of the whole epipelagic layer microbiome [31]. From the same kind of sample, we have abundant information from previous metagenomic analysis [31-34]. We applied the two $3^{\text {rd }}$ generation sequencing platforms and analyzed the results pre and post assembly. We propose a specific pipeline based on CCS processing of the raw PacBio reads and their ulterior assembly to retrieve more useful information directly from the individual LRs, and much more complete MAGs than SR might allow. 


\section{$101 \quad$ RESULTS AND DISCUSSION}

\section{LR platforms output}

103 A comparison of the metagenomic datasets generated by the three sequencing

104 platforms is shown in Table 1. It is already apparent that with equivalent costs, one

105 PacBio run produced 18 and 320 times more raw data (Gb) than Illumina or Nanopore 106 sequencing, respectively. In addition, the largest sequenced read of ca. $448.5 \mathrm{~Kb}$ was

107 also achieved with PacBio, whereas Nanopore produced the longest read of $35.5 \mathrm{~Kb}$.

108 However, when comparing the average read size, numbers were not so different, with

1095.4 and $4.1 \mathrm{~Kb}$ for PacBio and Nanopore, respectively, i.e. extra long-reads are

110 infrequent. Nanopore raw reads are known to have a significant error-rate in their

111 sequences. Inspection of the dataset showed a variation comprised between 80 and

$11297 \%$ read accuracy (Figure S1). Actually, 58\% of the total sequence generated (851.5

$113 \mathrm{Mb}$ ) had an error rate lower than $10 \%$ and only $98 \mathrm{Mb}$ lower than $5 \%$. Also, we

114 detected a shift in the GC content of the corrected sequences compared to the raw

115 reads of all the other three methods (Figure S2) (28\%), indicating that error-correction

116 in Nanopore can significantly alter the relative abundance of the prokaryotic taxa

117 present in the sample. PacBio Sequel II does not provide the phred quality-score [35]

118 of the dataset [36]. Therefore, comparisons at this level are not feasible.

\begin{tabular}{|c|c|c|c|c|c|c|c|}
\hline \multirow{2}{*}{$\begin{array}{l}\text { Sequencing Technology } \\
\text { Read Type (Proccesing) }\end{array}$} & \multirow{2}{*}{$\begin{array}{c}\text { Illumina } \\
\text { (Nextseq } \\
2 \times 100 \mathrm{bp} \text { ) } \\
\text { Trimmed } \\
\text { Reads } \\
\end{array}$} & \multicolumn{2}{|c|}{$\begin{array}{l}\text { Oxford Nanopore (Minlon } \\
\text { Flow Cell, v9.4R) }\end{array}$} & \multicolumn{4}{|c|}{ PacBio Sequel II (8M SMRT Cell Run) } \\
\hline & & $\begin{array}{l}\text { Raw } \\
\text { Reads }\end{array}$ & Corrected Reads & Raw Reads & CCS5 & $\operatorname{ccs} 10$ & CCS15 \\
\hline \multicolumn{8}{|l|}{ Sequencing statistics: } \\
\hline \#Sequences (Millions) & 234.5 & 0.3 & 0.1 & 81.4 & 2.8 & 1.9 & 1.5 \\
\hline \#Nucleotides sequenced (Gb) & 23.4 & 1.4 & 0.4 & 439.6 & 15.4 & 10.1 & 7.6 \\
\hline Largest read size (bp) & 100 & 35,490 & 22,220 & 448,515 & 36,542 & 24,735 & 17,976 \\
\hline Average length read size (bp) & 99.6 & $4,117.9$ & $3,148.2$ & $5,401.6$ & $5,422.4$ & $5,190.7$ & $4,968.8$ \\
\hline \multicolumn{8}{|l|}{ Predicted proteins (Reads > $1 \mathrm{~Kb}$ ): } \\
\hline \#Proteins (Millions) & -- & 1.3 & 1.5 & 368.1 & 21.6 & 12.2 & 9.0 \\
\hline Average Protein Size (aa) & -- & 85.9 & 83.2 & 90.4 & 195.1 & 241.5 & 248.4 \\
\hline Proteins / Mb Sequenced & -- & 937.3 & $3,907.9$ & 837.3 & $1,407.1$ & $1,212.8$ & $1,184.4$ \\
\hline
\end{tabular}


120 Table 1. Summary statistics of the short-read and long-read sequencing technologies and protein-

121 encoded genes retrieved from reads.

123 However, to guaranty a low error rate of PacBio, we applied the software "Highly

124 Accurate Single-Molecule Consensus Reads" (CCS reads) [17]. The algorithm selects

125 DNA tracts that are re-sequenced up to the number provided (5, 10, or 15 times).

126 These numbers theoretically achieve $99,99.9$ and $99.95 \%$ base call accuracy,

127 respectively. Thus, for example, the total PacBio sequence generated decreased from 439.63 $\mathrm{Gb}$ (raw) to $7.63 \mathrm{~Gb}$ (CCS15) (Table 1). To assess the read accuracy, we assumed that erroneous nucleotides would lead to an increase of stop codons in the

130 predicted proteins and could be measured by the decrease of their average protein size. As seen in Table 1 PacBio raw reads had error rates similar to those of Nanopore

132 (average protein size 90.4 and 85.9 amino acids respectively) while CCS15 provided an average protein size of 248.4 amino acids, much closer to the values of the two

134 dominant microbes in these waters (302.5 or 255 found for Ca. Pelagibacter

135 HTCC7211 and Prochlorococcus marinus MED4 pure culture genomes, respectively).

136 Thus, we have concluded that the quality of CCS15 is enough to get a reliable picture

137 of the genes present in the sample. Contrastingly, Nanopore does not seem suitable

138 for cellular (as opposed to viral $[18,37]$ ) metagenomic analysis. Henceforth, we will

139 focus the analysis on the PacBio results that we will refer to as long-reads (LR) and

140 Illumina Nextseq as short-reads (SR).

142 Taxonomic profiling of samples by metagenomic rRNA operons

143 The community structure of a metagenomic sample is one of the most basic pieces of 144 information about a microbial assemblage and can be assessed by multiple 145 approaches $[38,39]$. One of the most reliable is the isolation of SRs that have hits to 
$14616 \mathrm{~S}$ rRNA genes and use their huge databases to affiliate the sequences and thus the

147 microbes behind. This can be done with the individual SRs or with SR assembled

148 rRNA genes [40], although assembly of these highly conserved sequences is not very

149 reliable. In the case of LR sequencing, complete (or nearly so) rRNA genes and even

150 operons can be retrieved within a single contig making assembly superfluous [21,41].

151 We have extracted and compared 16S rRNA gene fragments to check whether LRs can improve the taxonomic affiliation. Thus, we were able to extract 9,763 16S rRNA sequences from LR CCS15 of our sample (average length: 1,207 bp; $0.34 \%$ of total LRs), and 20,564 SRs (average length: $95 \mathrm{bp} ; 0.22 \%$ of total SRs). These sequences were classified against the SILVA database (Table S1), The community structure derived from LRs was nearly identical to the one obtained from SRs down the level of families (Figure 1A and Table S1), with only a significant exception in Cyanobacteria, that were overrepresented in the SR dataset, $9.1 \%$ compared to $6.9 \%$ in the LRs. However, the availably of longer gene fragments with LRs improves the 16S rRNA

160 classification, decreasing the number of reads that were not classified to any specific 161 phylum (0.4\% LR versus $1.3 \%$ SR, ) or could not be ascribed to lower-level taxa 162 (4.3\% that only reached the class level Alphaproteobacteria with SRs versus only $1.3 \%$ LRs), (Figure 1A and Table S1). These results indicate a better resolution microbial classification of LRs. More importantly, LRs have the potential to uncover complete 16S rRNA sequences from "dark matter" [2] microbes with a higher level of resolution and reliability avoiding potential assembly artifacts.

167 Furthermore, other useful identifiers within the ribosomal operon, including hypervariable regions such as the internal transcribed spacers (ITS), could be 169 retrieved within a single contig $[42,43]$. These allow a precise community structure 170 determination that includes ecotypes or even strains. As an example, the ITS tree for 
171 the picocyanobacterial retrieved in our sample is shown in Figure 1B. We considered

172 only complete ITS sequences (both $16 \mathrm{~S}$ and $23 \mathrm{~S}$ genes had to be present in the same

173 read). 170 ITSs could be extracted, of which $68 \%$ were classified as Synechococcus.

174 Within this genus, clades IV (69 ITS) and I (24 ITS) were the most dominant in the

175 sample (Figure 1C). These clades have been detected before in cold coastal waters

$176[44,45]$ so their presence in our mixed winter sample was expected. Along similar lines,

177 two Prochlorococcus ecotypes dominated the sample. Forty out of 54 ITSs were

178 assigned to the High-Light I (HL-I) ecotype, while only 14 sequences grouped within

179 the Low-Light I (LL-I) ecotype (Figure 1C). These results fit well with genome

180 recruitment data using pure cultures or MAGs of the different ecotypes as reference

181 carried out on in this and similar samples collected different years, seasons, and

182 depths [31,32], supporting the reliability of the LR ITS data.

A
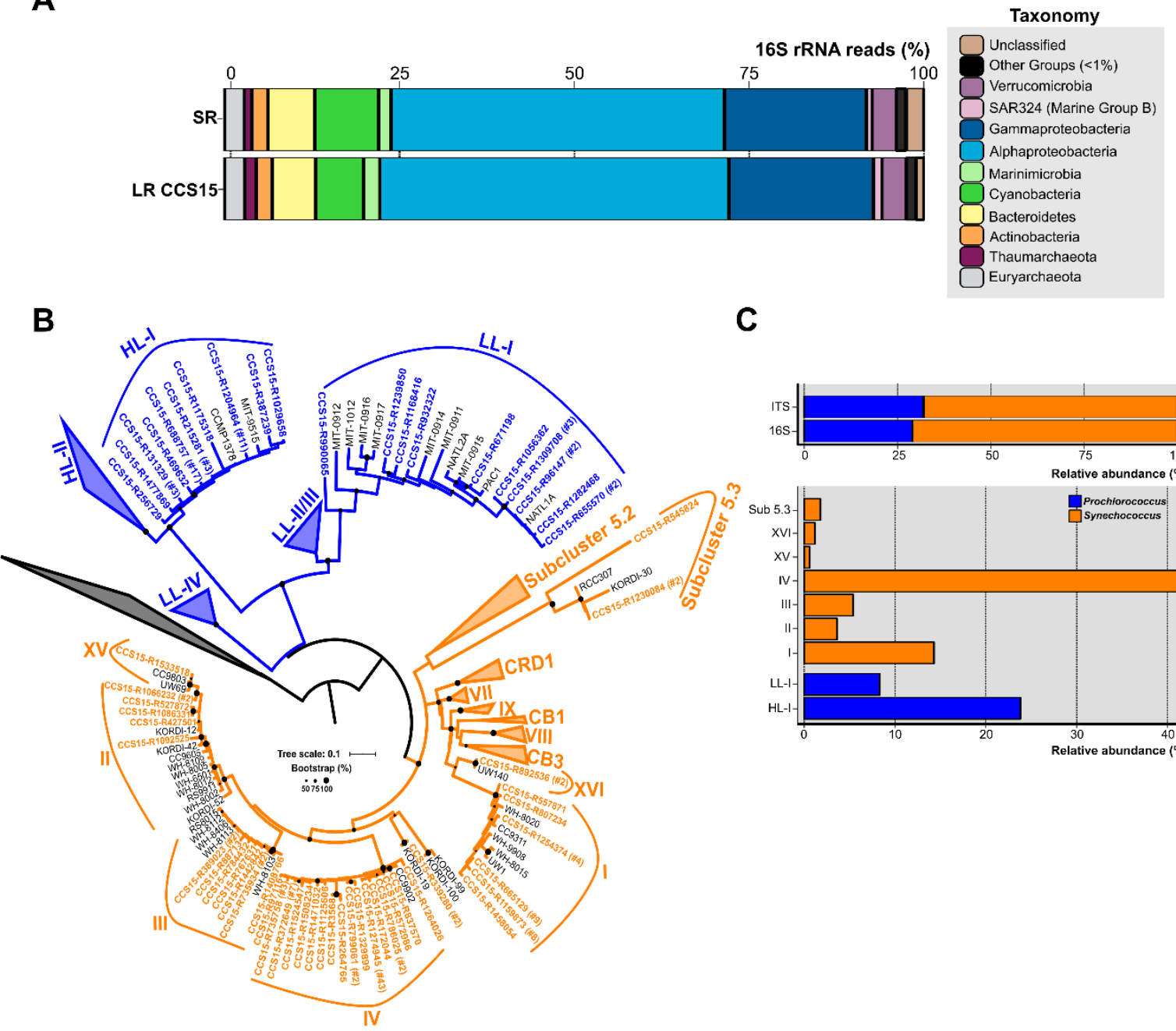

C
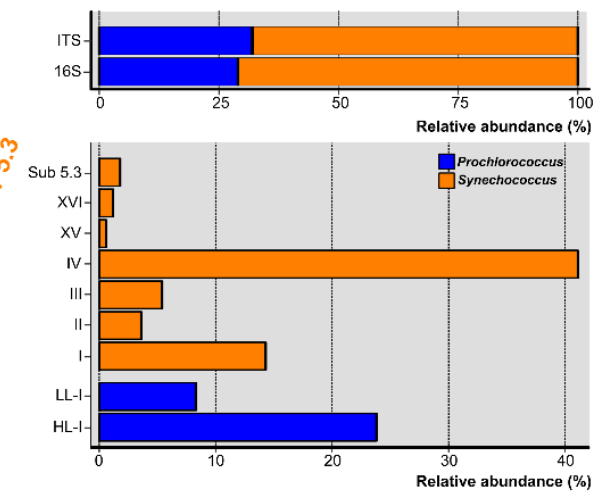
183 Figure 1. A. Phylum-level composition based on 16S rRNA gene fragments of Illumina (SR) and PacBio

184 CCS15 metagenomic reads. The phylum Proteobacteria was divided into its class-level classification.

185 Only those groups with abundance values larger than $1 \%$ are shown. B. Maximum-likelihood

186 phylogenetic tree of the 16S-23S rRNA genes ITS extracted from CCS15 reads classified by 16S rRNA

187 as Cyanobacteria. Sequences are coloured according to their affiliation to Prochlorococcus (blue) or

188 Synechococcus (orange). In order to simplify the tree, nucleotide sequences were dereplicated at $99 \%$

189 identity. Numbers between brackets indicate the number of sequences that clustered at this level to a

190 given CCS15 read (in bold). ITS from reference isolates are also shown (black). C. Upper panel, relative

191 abundance of Synechococcus and Prochlorococcus identified from ITS or 16S rRNA sequences in the

192 CCS15 reads. Bottom panel, ITS classified into subclades and ecotypes.

Metagenomic assembly with LR

195 Still, the possibility to retrieve complete (or nearly so) genomes from metagenomes

196 (MAGs) is highly informative for understanding uncultivated microbes. In principle, the

application of LR to a complex sample could improve metagenomic assembly by simplifying the leap across repeats that hamper SRs assembly. However, the choice of an assembler for metagenomic projects is not trivial. To gauge the applicability of different programs we have to consider also the possibility of a hybrid assembly to

201 take advantage of the high coverage and low error rate of SRs. We selected two specific assemblers for LRs (based on overlaps, Canu or Bruijn Graphs, MetaFlye) and one that is hybrid and can combine SRs and LRs (see methods). Only assembled contigs larger than $5 \mathrm{~Kb}$ have been further considered.

In a first approach, five subsets of LRs (before CCS processing) larger than $7 \mathrm{~Kb}$ were assembled (Table S2). Assembly results by metaFlye and Canu were positively correlated (close to linear, data not shown) with the sequencing effort. The largest contig size also followed this pattern, while the average contig size was not variable within the range considered. Besides, these assemblers resulted in a low number of

210 proteins per $\mathrm{Mb}$ and small average protein size, indicating low-quality assembly (Table

211 S2). Conversely, metaSPAdes (hybrid assembly LR and SR) showed that the effect of 
assembling larger amounts of PacBio raw reads did not have a linear trend in the

213 resulting assembly. Besides, due to the inclusion of SRs in the assembly process, at

214 lower coverage values, metaSPAdes assembly was larger than the other two

215 assemblers. Furthermore, given the restricted use of LRs in the hybrid assembly (it

216 mainly comes from the SRs), the high error rate of LRs did not affect the quality

217 (average size) of the resulting assembled proteins (Table S2). Thus, metaSPAdes

218 appears as the best option for assembly of LR as long as an SR dataset is also

219 available.

220 We also evaluated the effect of increasing steps of CCS in the assembly by the three

221 software packages compared to SRs IDBA assembly (SRa). Regardless of the CCS steps $(5,10$, or 15$)$, the resulting assembly outperformed IDBA SR (Figure 2A). The largest contig was achieved with metaSPAdes CCS5, 2.6 Mb long, one order of magnitude higher than the one achieved with SRa $(275 \mathrm{~Kb})$. Besides, the average genome size was also seven times higher with metaSPAdes CCS5 (Figure 2A). Although it yielded a lower assembled output than the other two methods, the contigs had smaller average protein sizes (Figure 2B lower panel) nullifying the advantage.

228 Therefore, the best assembly results in terms of assembly size and, particularly, reliability were achieved using metaSPAdes with the pool of CCS15 LRs (Figure 2A and B). Unfortunately, we lose the longest fragments obtained in the CCS5 assembly,

231 likely due to a decrease in the coverage (Table 1). To validate the assembly of

232 metaSPAdes CCS15 (Figure 2C) we have compared the large-scale taxonomic affiliation of the contigs with those of the SRa (Figure 2C). All phyla were recovered

234 (and in similar proportions) by both methods and only small numerical differences were 235 found confirming that no major bias (at least not different from those that might 
A
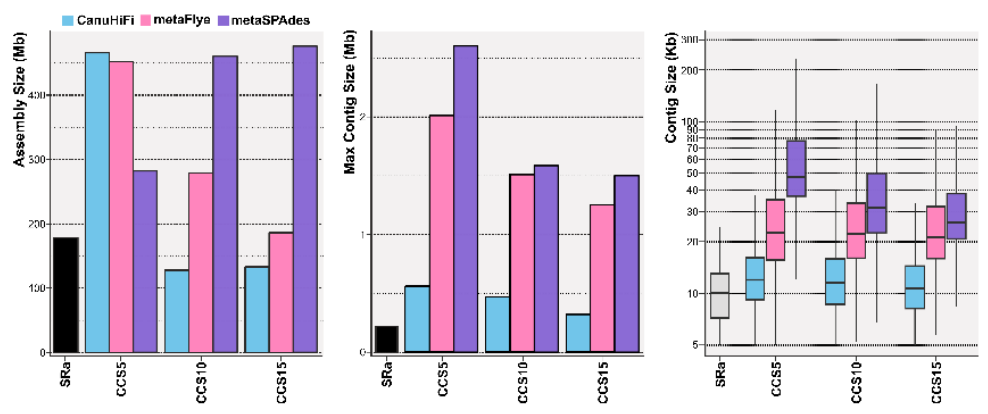

C
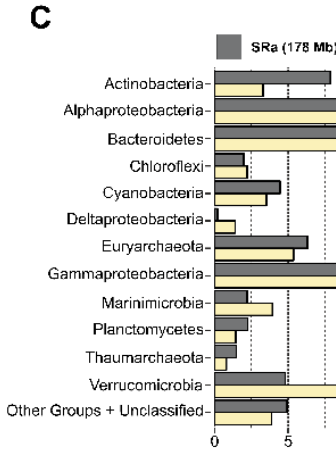

238
B

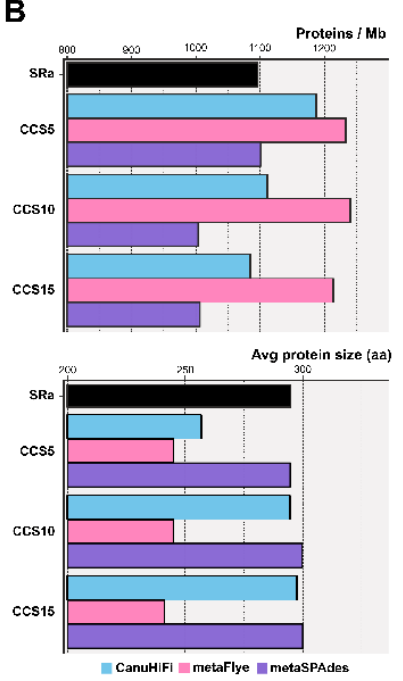

D

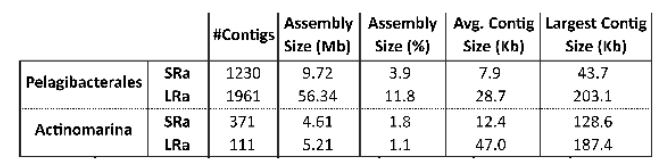

Figure 2. A. Bar and box plots indicating the total assembly size, maximum contig length and contig size distribution for the Illumina (SR) and PacBio CCS5, CCS10 and CCS15 assemblies. CCS reads were assembled with CanuHiFi, metaFlye and metaSPAdes (blue, pink and violet bars, respectively). B. Similar to A, but representing the number of predicted proteins assembled per Megabase (upper panel) and the average protein size (lower panel). C. Taxonomic classification at the level of phylum of the resulting Illumina (SRa) and PacBio CCS15 (LRa) assemblies. The phylum Proteobacteria was divided into its class-level classification. D. Summary of the assembly statistics for contigs classified as Ca. Pelagibacterales and Ca. Actinomarinales.

One major problem of the classical MAG approach is its proven low yield of some of the most prevalent members of the community. A very prominent example in the marine environment is the Pelagibacterales [46]. Despite their dominance in open epipelagic marine waters [46], the numbers of MAGs retrieved in metagenomic studies are relatively small, with only 34 MAGs (medium quality, $>50 \%$ complete and $<5 \%$ contaminated) available presently in public repositories [7]. Another example is Ca. 
254 Actinomarinales [47], a cosmopolitan marine actinobacterium that for accounts up to $5 \%$ of the prokaryotic community and has only seven MAGs available [48]. The reasons for this anomaly are unclear but the most likely explanation points to the high level of sequence microdiversity characteristic of these microbes. Here, the use of LR metagenomics improved considerably the assembly of both microbes (Figure 2D). LRa achieved a better assembly size, as in Pelagibacterales, with $\sim 6$ times more data with LRa than SRa, and longer contigs that might help the recovery of complete (or nearly so) MAGs.

\section{Recovery of novel genes}

264 One of the major levers of metagenomics is its capacity to identify new proteins that alone can provide important insights into the ecology of the sample and often is capable to associate the activities to certain microbial groups. In any case, the expression in surrogate vectors, allows the use of the recovered proteins for structural or biotechnological studies. LRs can span complete genes (or operons) and, therefore, avoid the SR assembly step, which is greatly biased by the choice of the assembler and gene calling $[49,50]$ together with the (micro)diversity and abundance of prokaryotes in the sample [51]. A recent study [52] evaluated how the application of high-throughput metagenomic sequencing has severely improved the catalog of marine microbial genes. Large metagenomic studies, such as Tara Oceans [30],

274 GEOTRACES [53] and Malaspina [54], sequenced and assembled hundreds of marine SR datasets at different years, seasons, latitudes, and depths. They have retrieved ca. 50M non-redundant proteins [52]. Yet, when this number is normalized by the sequencing effort $(4.8,4.8$ and $52.1 \mathrm{M}$ non-redundant proteins/Tb, respectively 278 [52]), the numbers become smaller than those retrieved by the Global Ocean Sampling 
by cloning and Sanger sequencing (GOS [55]) (624 M /Tb [52]). In our work, LR sequencing of just one metagenomic sample yielded 3.6 M non-redundant proteins, what can be extrapolated to $473.7 \mathrm{M} / \mathrm{Tb}$, very close to the GOS numbers, but with a largely diminished cost/person-power investment, and better yield of reconstructed genomes (see below) and gene clusters. To assess further the differential capability to recover novel proteins by LR metagenomics we have selected to search in our single metagenomic sample for four common objectives of screenings for biotechnologically relevant proteins or gene clusters: rhodopsins, polyketide synthases (PKS), and CRISPR systems.

288 One of the best examples of the biotechnological harvest of metagenomics has been 289 the retrieval of a vast diversity of retinal proteins (rhodopsins) [56-58] critical for the development of optogenetics, a technology with remarkable potential in neurobiology and medicine $[59,60]$. The photic zone of the ocean is the quintessential habitat to screen for the diversity of rhodopsins and already many have been retrieved by SR assembly metagenomics [61,62]. Remarkably, the largest numbers of rhodopsins

294 (>200 amino acids, clustered at $90 \%$ amino acid identity) were found in the individual LRa (330 rhodopsin genes/Gb assembled) (Figure 3A). However, considering the sequencing effort to assemble (31 Gb, sum of SR and LR CCS15), this number decreases down to 5 rhodopsins / Gb, smaller than the LR output (50 rhodopsins / $\mathrm{Gb).} \mathrm{This} \mathrm{result} \mathrm{illustrates} \mathrm{the} \mathrm{performance} \mathrm{of} \mathrm{LR} \mathrm{to} \mathrm{recover} \mathrm{novel} \mathrm{proteins} \mathrm{without} \mathrm{the}$ 299 biased assembly step. Besides, a clustering at $>30 \%$ identity of all the rhodopsins retrieved with LR CCS15 (\#2858) and Tara assemblies (\#5887) resulted in 25 distinct protein clusters (data not shown), where twelve of them grouped sequences from both datasets. Eleven clusters had only sequences originating from Tara assemblies. Tara samples span different locations, depths, and seasons; thus, it was to be expected 
that its dataset contained a higher diversity of rhodopsins than our single sample.

305 Nonetheless, we could identify three novel rhodopsin clusters

306 without Tara representatives, indicating there is still room for the discovery of novel

307 rhodopsins through LR metagenomics. In fact, one of these three clusters contained

308 sequences similar ( $67 \%$ amino acid identity) to RubyACRs [63], a recently reported

309 anion channelrhodopsin with promising implications in optogenetics.

310 From a biotechnological point of view, one of the most important natural products is

311 polyketide synthases [64,65]. They are large proteins and often require other

312 accompanying genes to be functional. Besides, they tend to be located at the flexible

313 genome that as mentioned before assembles poorly in SR metagenomes. The total

314 number of PKS type 1 (long and modular proteins) was similar to the three datasets

315 (LRs, SRa, and LRa). The other two types, type 3 (smaller proteins that work only with

316 the complement of the other members of the cluster) and heterocyst glycolipid

317 synthase PKS (cyanobacterial PKSs) were better recovered by LRa (Figure 3A).

318 Actually, in LR individual reads there were type 1 complete clusters. One of them was

$319100 \%$ similar to the 1-heptadecene biosynthetic gene cluster from Cyanothece sp.

320 PCC 7822 [66]. Some type 3 PKS (mostly chalcone synthases from Synechococcus),

321 were also recovered complete (data not shown). Thus, as in the case of rhodopsins

322 assembly seems rather redundant for LR screening for PKSs.

323 Although CRISPR systems are very scarce in seawater, these systems are also often

324 screened for and described from metagenomic datasets [67]. They form large clusters of Cas (CRISPR associated) proteins together with long stretches of tandem repeats

326 [68]. We could find four LRs containing both Cas proteins and complete CRISPR

327 arrays (Figure 3B); this number increased up to 15 if we included sequences with no

328 Cas proteins but with an evidence value $\geq 4$ following CRISPRdetect [69]. A 
comprehensive search of the spacers in a custom database containing metagenomes, viromes, and reference viral sequences (see methods) showed that $28 \%$ of them were positively affiliated to viral sequences (Figure 3B). LRs CCS15-R1170264 and CCS15-R276795 represent two CRISPR arrays that affiliated with two different and uncultured Planctomycetes/Verrucomicrobia bacteria (Figure 3C). However, their spacers indicated two different geographic distributions. Spacers in CCS15-R1170264 matched several sequences recovered from different locations, indicating a sea.

A
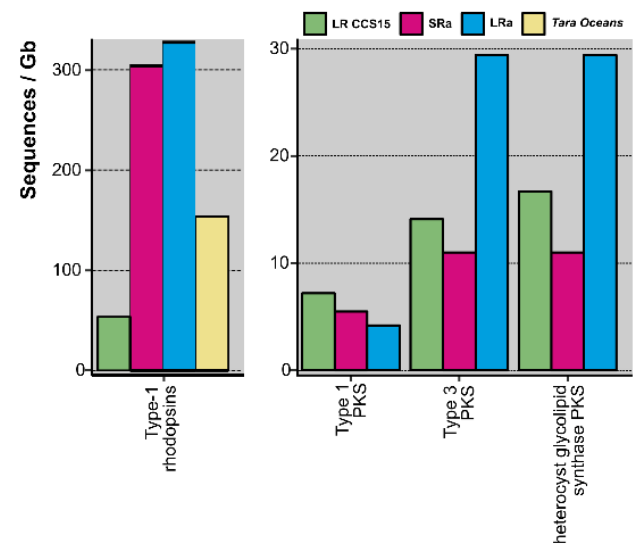

C

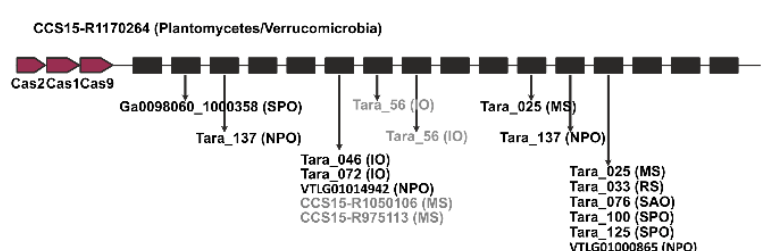

B
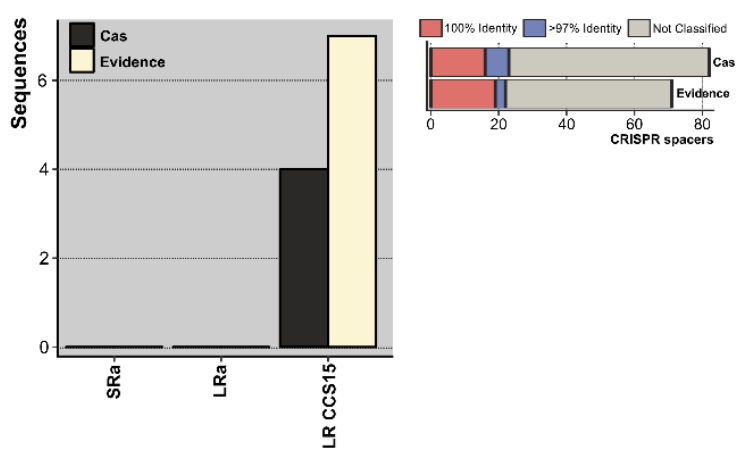

CCS15-R276795 (PlantomycetesNerrucomicrobia)

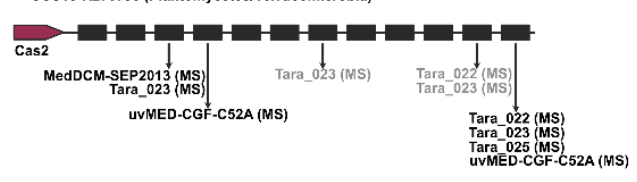

Figure 3. A. Number of type-1 rhodopsins and polyketide synthases (PKS) (left and right panels, respectively), retrieved from the LR CCS15 reads (green bar), from SRa and LRa assemblies (red and blue blars, respectively) and from Tara Oceans assemblies (yellow bar). Hits are normalized by the size of the database (in Gigabases).

B. Left panel, number of sequences (LR CCS15, and SRa and LRa contigs) containing CRISPR and Cas proteins (coloured in black) or only a CRISPR array with an evidence score $\geq 4$ (light yellow bar). Right panel, number of CRISPR spacers classified at 100 and $>97 \%$ identity. Sequences failing the $97 \%$ identity threshold or not matching to the database are 
grouped into the "not-classified" bar. C. Two examples of metagenomic reads having a CRISPR array and Cas proteins. Sequences in black and grey represent 100 and $>97 \%$ identity hits. Between brackets the isolation source of the hit: Mediterranean Sea (MS); Red Sea (RS); Indian Sea (IO), North and South Atlantic Oceans (NAO, SAO); and North and South Pacific Oceans (NPO, SPO).

\section{Recovery of genomes}

354 To assess the efficiency of MAG retrieval by LRa we extracted 77 MAGs (>50\% completeness, $>5 \%$ contamination). This figure is rather small compared to other similar studies carried out by SRa. However, when corrected for the amount of processed sequence (CCS15 only) the ratio is higher than in similar studies carried out with similar samples by SRa, as well as the average contig size and the degree of completeness (Figure 4). To compare the MAG reconstruction carried out by both approaches, we selected 31 MAGs retrieved in the previous SRa works carried out in our laboratory $[31,34]$ and that had $>99.5 \%$ average nucleotide identity (ANI) to MAGs and the average contig size was 4 times larger, what clearly turns the balance in favor of LRa to get high-quality reliable MAGs.

A

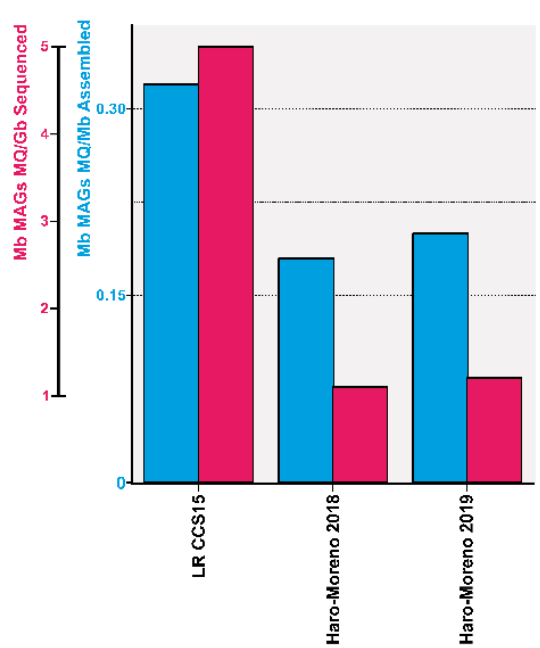

B
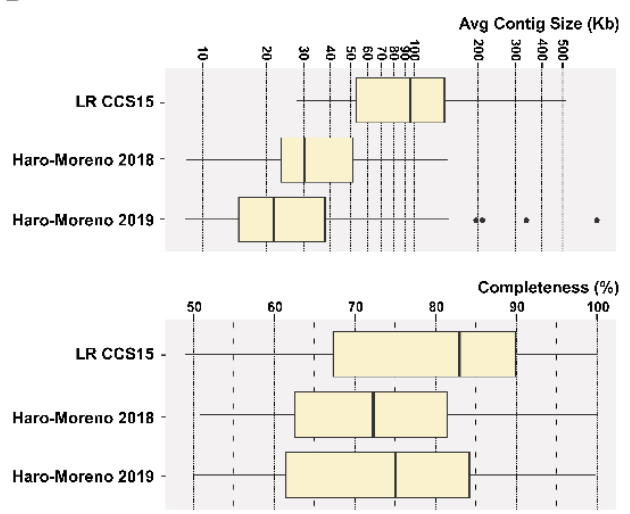
Figure 4. A. Contribution of medium-quality MAGs ( $>50 \%$ complete and $<5 \%$ contamination) recovered in this study (LR CCS15) and from two previous reports from the same location in the Mediterranean Sea, Haro-Moreno et al. 2018 [31] and Haro-Moreno et al. 2019 [34]. Values are normalized by the assembly size and sequencing effort (blue and red bars, respectively). B. Box plots showing the average contig size and the degree of completeness of MAGs described in A.

Visual inspection of the LRa MAGs indicated very complete and easily closable collections of contigs. To objectively compare the completion of MAGs generated by both approaches we could identify two microbial genomes that are derived from pure cultures and were retrieved also in LRa and SRa, and compared the assemblies with the reference genomes (assumed to be complete) (Figure 5). One of them was a genome similar (ANI 97\%) to the cyanobacterium Prochlorococcus marinus MED4 (high-light-adapted ecotype) [72], one of the most abundant microbes in our kind of sample (30 RPKG in the sample analyzed here). The SRa was only $2 \%$ complete (estimated by CheckM) with 6 small contigs among which the longest was $34 \mathrm{~kb}$ (Figure 5A). The LRa MAG covered nearly the complete pure culture genome, with only eight contigs, the longest $608 \mathrm{~kb}$, and with more than $98 \%$ of the pure culture genome (Figure 5A). Gaps were found mostly at the location of the known major flexible genomic island of this microbe [73] particularly, Gl4 that codes for the O-chain polysaccharide [74]. We also reconstructed by both assemblies a relative $(93.4 \% \mathrm{ANI})$ of the Thaumarchaeon Ca. Nitrosomarinus catalina SPOT01 [75] by LRa and SRa. LRa produced only three contigs, the longest being $1 \mathrm{Mb}$ with a $99 \%$ completeness based on checkM (data not shown). The comparison with the reference genome Ca. Nitrosomarinus catalina SPOT01 showed that two regions were not covered (Figure

391 5A). One largely corresponds to a prophage that might not be present in our local 392 relative, and the other again a genomic island putatively involved in the synthesis of a polysaccharide equivalent to GI4 in MED4 [73]. 
394 It has been established that there are two main categories of flexible genomic islands

395 (fGls) in prokaryotic genomes: i) replacement fGls are involved in synthesizing the

396 outer glycosidic envelope of the cells (such as the O-chain in Gram-negatives) [76]

397 that varies between closely related strains, and ii) additive fGls (such as integrons)

398 that vary more gradually by replacement of relatively small gene cassettes that appear

399 among other gene clusters conserved between strains. One of the problems inherent

400 to the assembly of short reads is the failure to assemble fGIs in prokaryotic genomes.

401 The reasons are multifactorial, i) SRa contigs belonging to fGls tend to bin separately

402 due to variations in genomic parameters, ii) they are less abundant since are only

403 harbored by some lineages within the population, and finally iii) replacement fGls tend

404 to be surrounded by highly variable (if conserved) genes that are followed by totally

405 divergent sequences [77]. All these scenarios make assembly algorithms highly

406 inefficient in retrieving fGls. This is a major setback since many genes of

407 biotechnological potential are found within flexible genomic islands. Furthermore,

408 many key ecological functions such as transporters, degradation of resilient

409 compounds, virulence factors and many others are also found in these genomic

410 regions $[78,79]$. That the long replacement GI4 do not assemble in the MED4 LRa

411 MAG was to be expected given the high diversity of very polyclonal microbes such as

412 Prochlorococcus [80] and the length of this specific fGI involved in the synthesis of the

413 O-chain polysaccharide $[81,82]$. The presence of multiple (and long) versions of GI4

414 might disorient the assembler that has many possibilities to continue the contig. On

415 the other hand, the small island present in the reconstructed $\mathrm{Ca}$. Nitrosomarinus MAG

416 (Figure 5B) might be short enough to appear in one single read and it did appear in

417 the MAG and other identical fragments recovered as LRs. 


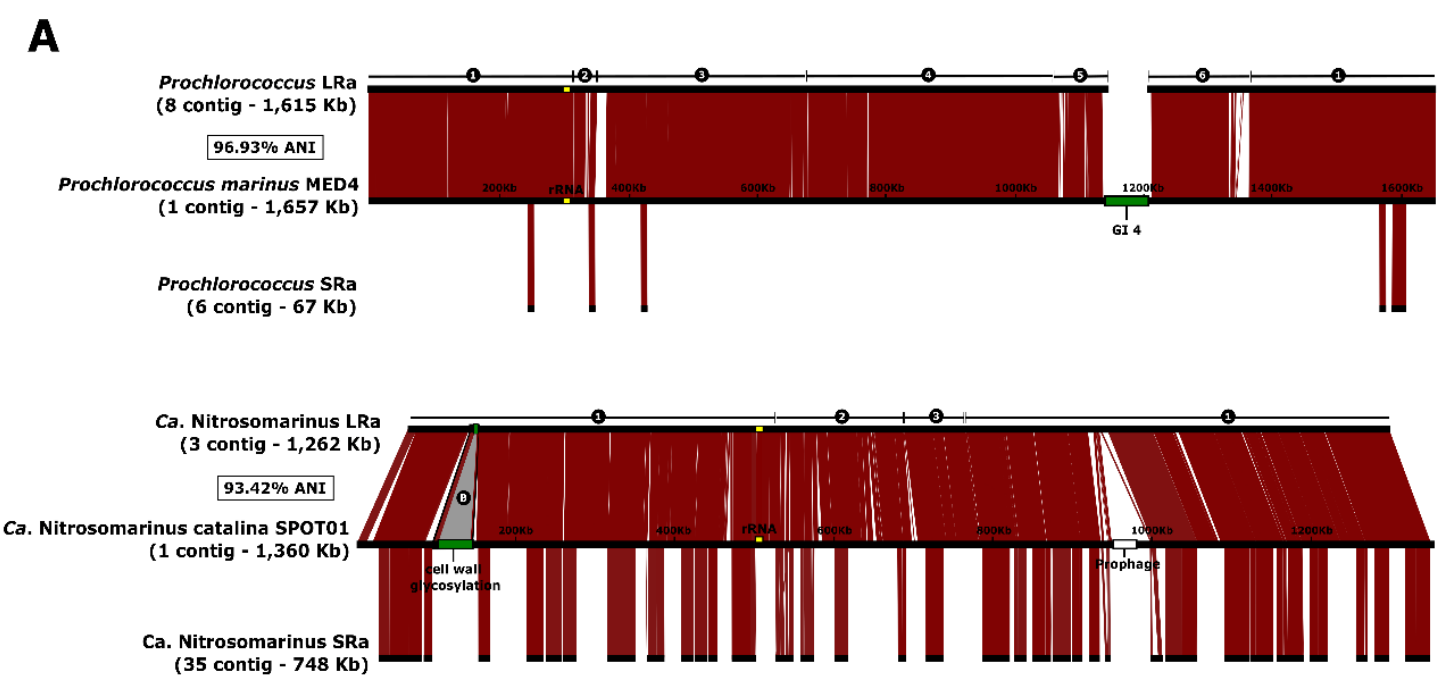

B

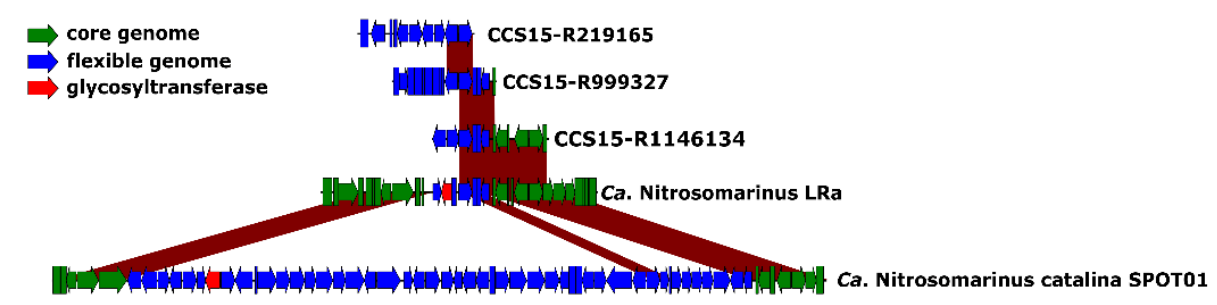

420 Figure 5. A. Alignment of the reference genomes Prochlorococcus marinus MED4 and Ca. 421 Nitrosomarinus catalina SPOT01 against the reconstructed MAGs from LR CCS15 (above) and SR (below). Number of contigs, and ANI of the LR CCS15 MAGs and the reference genomes indicated to the left. GI4 of $P$. marinus coding for O-chain polysaccharide synthesis [74] and a putative cell was glycosylation cluster in of $\mathrm{Ca}$. N. catalina are in green. B. Magnified B region as labelled in A indicating also some syntenic fragments found among CCS15 reads.

To assess the improvement in the retrieval of flexible genomic islands by LRa, we in more detail one marker that is usually found in these islands: arrays of glycosyl transferases (GT). Genome analyses have demonstrated that in this $\mathrm{Gl}$ there is an accumulation of GTs and, therefore, these genes are a good indicator for the recovery of such gene clusters. We have considered only fragments between $5 \mathrm{~Kb}$ encoding 432 for at least 5 GTs in a window of maximum 20 genes as putative parts of these 433 glycosylation islands. Indeed, LRa recovered more than $300 \mathrm{GT} / \mathrm{Gb}$ while SRa only 434 found 100. The presence of more GTs in the LRa can be explained by the short 
436 flexible Gls that contain only small differential cassettes, with conserved clusters

437 alternating with variable ones, that can be straddled by individual reads, would be

438 recovered much more efficiently, which would explain the increase of typical additive

439 Gls components such as the PKS or CRISPR (see above).

\section{CONCLUSIONS}

\section{Is LR metagenomics the next step?}

443 The answer appears to be largely affirmative. Although SR approaches might be used

444 to complement for recruitment of known genomes or to improve assembly, for most

445 purposes LR sequencing is much more rewarding both in terms of the amount and 446 quality of the information. It only requires slightly more environmental DNA and of 447 better quality (more care should be taken to avoid too much fragmentation of the DNA 448 in the sample) and the cost per properly annotated gene is significantly lower. 449 Furthermore, it allows a first glimpse at the flexible genome of many microbes in which 450 a wealth of potentially useful biotechnology might be hidden. It might complement 451 SAGs and MAGs to get complete and reliable genomes of the many novel groups that 452 have been uncovered during the last decade, improve their annotation and their representation in databases and eventually lead to a more realistic picture of the real diversity of microbes. The enhanced recovery of the flexible genome would provide a better understanding of their ecological features and their potential applications. Last but not least, the intricacy of natural populations of bacteria could be analyzed in detail providing a glimpse at microbial evolution in action. 


\section{MATERIAL AND METHODS}

\section{Sampling, processing, and sequencing}

463 Samples from two different depths (20 and $40 \mathrm{~m}$ ) were collected on February $15^{\text {th }} 2019$

464 from the epipelagic Mediterranean Sea, at 20 nautical miles off the coast of Alicante

465 (Spain) $\left(37.35361^{\circ} \mathrm{N}, 0.286194^{\circ} \mathrm{W}\right)$, during winter where the water column is mixed.

466 This location has been studied previously by metagenomic approaches $[31-34,70,83]$.

467 For each depth, $200 \mathrm{~L}$ were collected and filtered on board as described in [31]. Briefly, seawater samples were sequentially filtered through 20,5 and $0.22 \mu \mathrm{m}$ pore filter polycarbonate filters (Millipore). Water was directly pumped onto the series of filters to minimize the bottle effect. Filters were immediately frozen on dry ice and stored at -80 ${ }^{\circ} \mathrm{C}$ until processing.

DNA extraction was performed from the $0.22 \mu \mathrm{m}$ filter (free-living bacteria) following the phenol:chloroform extraction. Given the large amount of DNA needed for sequencing, DNA from the two samples $(20$ and $40 \mathrm{~m})$ was pooled together. Metagenomes were sequenced using Illumina Nextseq (100 bp, paired-end reads) (Macrogen, South Korea), and using Oxford Nanopore (one Minlon flowcell run, chemistry version 9.4R) and PacBio Sequel II (one 8M SMRT Cell Run, 30-hour movie) (Genomics Resource Center, University of Maryland, USA).

\section{Raw read filtering and assembly of metagenomic samples}

481 The quality of Illumina and Nanopore raw reads were examined with fastqc 482 (https://www.bioinformatics.babraham.ac.uk/projects/fastqc/) and nanopack [84].

483 PacBio Sequel II lacked a phred score. GC content in each sample was calculated 484 using the gecee program from the EMBOSS package [85]. Illumina raw reads were 485 trimmed with Trimmomatic [86] and assembled using IDBA-UD [87]. To improve the 
quality of the PacBio reads, we generated Highly Accurate Single-Molecule

487 Consensus Reads (CCS reads) using the CCS program of the SMRT-link package.

488 The minimum number of full-length subreads required to generate a CCS read was set to 5,10 and 15 (99, 99.9 and 99.95 base call accuracy, respectively. Nanopore and PacBio (raw and CCS reads) were assembled using the following assemblers: SPAdes [88] with the metagenome option and performing a co-assembly with the Illumina trimmed reads; Flye [89] with the metagenome option; and HiCanu [90]. MetaFlye is a de novo assembler that follows the classical de Bruijn graphs, although it allows for approximate sequence matches. Canu, on the other hand, applies an overlapping strategy for de novo assembly. Lastly, SPAdes needs both short-reads and long-reads to perform a hybrid assembly. However, in the latter case, LR are only used for gap closure and repeat resolution. Given that the error-rate in PacBio reads can be significantly improved, we used the resulting CCS15 reads as single reads in the hybrid assembly with SPAdes, and in that case, CCS reads can be used together with the Illumina reads for graph construction, gap closure and repeat resolution.

\section{Taxonomic and Functional annotation of PacBio reads and assemblies}

Prodigal [91] was used to predict genes from the assembled contigs retrieved from the individual assemblies of Illumina, Nanopore, and PacBio reads, as well as from the PacBio CCS reads. tRNA and rRNA genes were predicted using tRNAscan-SE [92] and barrnap (https://github.com/tseemann/barrnap), respectively. Predicted proteinencoded genes were taxonomical and functional annotated against the NCBI NR database using DIAMOND [93] and against COG [94] and TIGRFAM [95] using HMMscan [96]. 


\section{Taxonomic classification of metagenomic reads}

512 16S rRNA gene sequences were retrieved from Illumina and PacBio reads. Candidate

513 Illumina sequences in a subset of 20 million reads were extracted using USEARCH

514 [97] after an alignment against a non-redundant version of the SILVA database [98].

515 Sequences that matched to this database with an E-value $<10^{-5}$ were considered 516 potential 16S rRNA gene fragments. Then, ssu-align was used to identify true 517 sequences aligning these candidate sequences against archaeal and bacterial $16 S$ 518 rRNA hidden Markov models (HMM). For the long-read sequences, candidate 16S

519 rRNA sequences were extracted using barrnap from total PacBio CCS15 reads. The 520 resulting 16S rRNA sequences were classified using the sina algorithm [99] according

521 to the SILVA taxonomy database. Illumina sequences were only classified if the sequence identity was $\geq 80 \%$ and the alignment length $\geq 90 \mathrm{bp}$. Sequences failing these thresholds were discarded.

524 In addition, a total of 170 CCS15 contigs containing $16 S$ and $23 S$ rRNA genes of the 525 phylum Cyanobacteria were selected to perform an internal transcribed spacer (ITS) 526 phylogenetic tree, using the maximum-likelihood approach in iqtree [100], with 1000 bootstraps and the Jukes-Cantor model of substitution. Reference cyanobacterial ITS sequences were downloaded from the NCBI database.

\section{Genome reconstruction}

531 Assembled contigs longer than $5 \mathrm{~Kb}$ were assigned to a phyla classification if at least

$53250 \%$ of the genes shared the same best-hit taxonomy. Contigs failing this threshold 533 were grouped as unclassified. To bin the contigs into MAGs, their taxonomic affiliation

534 (including the unclassified) was used together with the principal component analysis 535 of tetranucleotide frequencies, GC content, and coverage values within this sample 
and several metagenomic samples described in previous studies from the

537 Mediterranean Sea [31,33,34,83]. Tetranucleotide frequencies were computed using wordfreq program in the EMBOSS package, and the principal component analysis was performed using the FactoMineR package [101]. Coverage values were calculated by

540 the alignment of metagenomic reads (in subsets of 20 million reads) against contigs using BLASTN [102] (99\% identity, > 50 bp alignment). Reads were normalized by the size of the contig in $\mathrm{Kb}$ and by the size of the metagenome in Gb (RPKGs). The degree of completeness and contamination of the resulting MAGs were estimated using

544 CheckM [103]. Average nucleotide identity (ANI) between MAGs and the reference genome was calculated using JSpecies software with default parameters [104].

Retrieval of relevant genes from the assemblies and the PacBio CCS reads.

548 Predicted protein sequences of contigs and PacBio CCS15 reads longer than $5 \mathrm{~Kb}$ were compared against several downloaded and custom datasets. HMM were 550 constructed using hmmbuild [96] for two curated databases of type-1 and type-3 551 rhodopsins. Searches were performed using hmmscan and only hits with a E-value < $55210^{-20}$ were considered. To remove redundant proteins, sequences were clustered from 100 to $30 \%$ identity using cd-hit [105]. Glycosyl transferases (GT) were retrieved using dbCAN [106] against the Carbohydrate-Active enZYmes (CAZy) database [107]. To consider GTs involved in the flexible genome, only genomic fragments with $\geq 5$ GTs and $\mathrm{E}$-values $<10^{-40}$ were analyzed.

Lastly, the bacterial version of the secondary metabolite biosynthesis database (antiSMASH) was used to identify and classify [108] polyketide synthases (PKS) gene clusters from contigs and PacBio CCS15 reads longer than $5 \mathrm{~Kb}$, and their taxonomic 
560 affiliation was based on consensus, that is $>70 \%$ of proteins encoded in a contig should share the same taxonomy (see above).

\section{Recovery and annotation of novel CRISPR-Cas systems.}

564 Sequences $\geq 5 \mathrm{~Kb}$ long were screened using CRISPR-detect [69] and CRISPR-cas

565 finder [109] tools. Only sequences matching in both methods and with an evidence 566 value $\geq 3$ were kept. The taxonomical affiliation of CCS reads and assembled contigs 567 was based on the annotation of coded proteins ( $>70 \%$ must share the same taxon).

568 To find the putative target, CRISPR spacers were aligned using the blastn-short 569 algorithm against nearly 200,000 phages collected and classified in [110]. Only 570 matches with $>97 \%$ identity and $100 \%$ alignment were considered. We also expanded 571 the search including numerous metagenomic and viromic assemblies recovered from 572 the Mediterranean Sea $[31,33,34,83]$ and other marine samples $[30,53]$.

\section{Data availability}

575 Metagenomic datasets have been submitted to NCBI SRA and are available under 576 BioProject accession number PRJNA674982 (Illumina reads: MedWinter-FEB2019-I 577 [SAMN16686071]; Nanopore reads: MedWinter-FEB2019-NP [SAMN16686072]; and 578 PacBio CCS reads: MedWinter-FEB2019-PBCCS15 [SAMN16686073]).

\section{$580 \quad$ ACKNOWLEDGEMENTS}

581 This work was supported by grants "VIREVO" CGL2016-76273-P [AEI/FEDER, EU]

582 (cofounded with FEDER funds) from the Spanish Ministerio de Economía, Industria y 583 Competitividad, "HIDRAS3" PROMETEU/2019/009 from Generalitat Valenciana and 584 5top100-program of the Ministry for Science and Education of Russia to FRV. JHM 
was supported by a PhD fellowship from the Spanish Ministerio de Economia y

\section{COMPETING INTERESTS}

591 The authors declare that they have no competing interests.

\section{AUTHORS' CONTRIBUTIONS}

594 The work was conceived by FRV. The analysis was carried out by JHM, MLP, and

595 FRV. The manuscript was written by FRV, JHM, and MLP. All authors read and approved the final version.

\section{REFERENCES}

600 1. Parks DH, Rinke C, Chuvochina M, Chaumeil P-A, Woodcroft BJ, Evans PN, et al.

601 Recovery of nearly 8,000 metagenome-assembled genomes substantially expands

602 the tree of life. Nat Microbiol. Springer US; 2017;903:1-10.

603 2. Castelle CJ, Banfield JF. Major New Microbial Groups Expand Diversity and Alter

604 our Understanding of the Tree of Life. Cell. 2018. p. 1181-97.

605 3. Sharon I, Banfield JF. Genomes from metagenomics. Science (80- ). American

606 Association for the Advancement of Science; 2013;342:1057-8.

607 4. Hugerth LW, Larsson J, Alneberg J, Lindh M V, Legrand C, Pinhassi J, et al.

608 Metagenome-assembled genomes uncover a global brackish microbiome. Genome

609 Biol. Genome Biology; 2015;16:279. 
610 5. Rinke C, Schwientek P, Sczyrba A, Ivanova NN, Anderson IJ, Cheng J-F, et al.

611 Insights into the phylogeny and coding potential of microbial dark matter. Nature.

612 Nature Publishing Group; 2013;499:431-7.

613 6. Chen LX, Anantharaman K, Shaiber A, Murat Eren A, Banfield JF. Accurate and

614 complete genomes from metagenomes. Genome Res. Cold Spring Harbor

615 Laboratory Press; 2020;30:315-33.

616 7. Haro-Moreno JM, Rodriguez-Valera F, Rosselli R, Martinez-Hernandez F, Roda-

617 Garcia JJ, Gomez ML, et al. Ecogenomics of the SAR11 clade. Environ Microbiol.

618 John Wiley \& Sons, Ltd; 2020;22:1748-63.

619 8. Rodriguez-Valera F, Martin-Cuadrado A-B, Rodriguez-Brito B, Pasić L, Thingstad

620 TF, Rohwer F, et al. Explaining microbial population genomics through phage

621 predation. Nat Rev Microbiol. 2009;7:828-36.

622 9. Bowers RM, Kyrpides NC, Stepanauskas R, Harmon-Smith M, Doud D, Reddy

623 TBK, et al. Minimum information about a single amplified genome (MISAG) and a

624 metagenome-assembled genome (MIMAG) of bacteria and archaea. Nat.

625 Biotechnol. Nature Publishing Group; 2017. p. 725-31.

626 10. Eid J, Fehr A, Gray J, Luong K, Lyle J, Otto G, et al. Real-time DNA sequencing

627 from single polymerase molecules. Science (80- ). American Association for the

628 Advancement of Science; 2009;323:133-8.

629 11. Clarke J, Wu H-C, Jayasinghe L, Patel A, Reid S, Bayley H. Continuous base

630 identification for single-molecule nanopore DNA sequencing. Nat Nanotechnol.

$6312009 ; 4: 265-70$.

632 12. Schadt EE, Turner S, Kasarskis A. A window into third-generation sequencing.

633 Hum Mol Genet. 2010;19:R227-40.

634 13. Goodwin S, McPherson JD, McCombie WR. Coming of age: ten years of next- 
generation sequencing technologies. Nat Rev Genet. 2016;17:333-51. purpose and place. Hum Mol Genet. 2018;27:R234-41.

15. Loman NJ, Quick J, Simpson JT. A complete bacterial genome assembled de novo using only nanopore sequencing data. Nat Methods. 2015;12:733-5. sequencing and assembly of a human genome with ultra-long reads. Nat Biotechnol.

17. Wenger AM, Peluso P, Rowell WJ, Chang P-C, Hall RJ, Concepcion GT, et al.

Accurate circular consensus long-read sequencing improves variant detection and assembly of a human genome. Nat Biotechnol. 2019;37:1155-62. free single-molecule sequencing recovers complete virus genomes from natural microbial communities. Genome Res. Cold Spring Harbor Laboratory Press; 2020;30:437-46.

19. Rhoads A, Au KF. PacBio Sequencing and Its Applications. Genomics, Proteomics Bioinforma. Elsevier; 2015. p. 278-89. the DNA sequencing data output [Internet]. PacBio Press Release. 2019 [cited 2020

654 Nov 6]. Available from: https://www.globenewswire.com/newsrelease/2019/04/24/1808652/0/en/Pacific-Biosciences-Launches-New-Sequel-II- 
661 Whole-Genome Shotgun Sequencing on Characterizing Reference Microbiomes.

662 iScience. Elsevier Inc.; 2020;23:101223.

23. Moss EL, Maghini DG, Bhatt AS. Complete, closed bacterial genomes from microbiomes using nanopore sequencing. Nat Biotechnol. 2020;38:701-7. sequencing of mock microbial community standards. Gigascience. 2019;8. based de novo assembly of low-complexity metagenome samples results in finished genomes and reveals insights into strain diversity and an active phage system. BMC Microbiol. 2019;19:143.

26. Xie H, Yang C, Sun Y, Igarashi Y, Jin T, Luo F. PacBio Long Reads Improve 2020. p. 1077.

27. Venter JC, Remington K, Heidelberg JF, Halpern AL, Rusch D, Eisen J a, et al.

675 Environmental Genome Sshotgun Sequencing of the Sargasso Sea. Science. 2004;304:66-74.

28. Thorpe J, Stewart C, Venter JECEC, Smith H, Nealson K, Eisen JA, et al. The

679 tropical Pacific. Moran NA, editor. PLoS Biol. Public Library of Science;

$680 \quad 2007 ; 5: 0398-431$.

29. Delong EF, Preston CM, Mincer T, Rich V, Hallam SJ, Frigaard N, et al.

682 Community Genomics among microbial assemblages in the Ocean's Interior.

683 Science (80- ). 2006;311:496-503.

684 30. Sunagawa S, Coelho LP, Chaffron S, Kultima JR, Labadie K, Salazar G, et al. 
685 Ocean plankton. Structure and function of the global ocean microbiome. Science.

$6862015 ; 348: 1261359$.

687 31. Haro-Moreno JM, López-Pérez M, de la Torre JR, Picazo A, Camacho A,

688 Rodriguez-Valera F. Fine metagenomic profile of the Mediterranean stratified and

689 mixed water columns revealed by assembly and recruitment. Microbiome. BioMed

690 Central; 2018;6:128.

691 32. Ghai R, Martin-Cuadrado A-BB, Molto AG, Heredia IG, Cabrera R, Martin J, et al.

692 Metagenome of the Mediterranean deep chlorophyll maximum studied by direct and

693 fosmid library 454 pyrosequencing. ISME J. 2010;4:1154-66.

694 33. López-Pérez M, Haro-Moreno JM, Gonzalez-Serrano R, Parras-Moltó M,

695 Rodriguez-Valera F. Genome diversity of marine phages recovered from

696 Mediterranean metagenomes: Size matters. Beja O, editor. PLoS Genet. Public

697 Library of Science; 2017;13:e1007018.

698 34. Haro-Moreno JM, Rodriguez-Valera F, López-Pérez M. Prokaryotic Population

699 Dynamics and Viral Predation in a Marine Succession Experiment Using

700 Metagenomics. Front Microbiol. Frontiers Media S.A.; 2019;10.

701 35. Ewing B, Green P. Base-calling of automated sequencer traces using phred. II.

702 Error probabilities. Genome Res. Cold Spring Harbor Laboratory Press; 1998;8:186-

70394.

704 36. Fukasawa Y, Ermini L, Wang H, Carty K, Cheung MS. LongQC: A quality control

705 tool for third generation sequencing long read data. G3 Genes, Genomes, Genet.

706 Genetics Society of America; 2020;10:1193-6.

707 37. Warwick-Dugdale J, Solonenko N, Moore K, Chittick L, Gregory AC, Allen MJ, et

708 al. Long-read viral metagenomics captures abundant and microdiverse viral

709 populations and their niche-defining genomic islands. PeerJ. PeerJ Inc.; 
2019;7:e6800.

711 38. Wood DE, Salzberg SL. Kraken: ultrafast metagenomic sequence classification

712 using exact alignments. Genome Biol. 2014;15:R46.

713 39. Huson DH, Beier S, Flade I, G??rska A, El-Hadidi M, Mitra S, et al. MEGAN

714 Community Edition - Interactive Exploration and Analysis of Large-Scale Microbiome

715 Sequencing Data. PLoS Comput Biol. 2016;12.

716 40. Yuan C, Lei J, Cole J, Sun Y. Reconstructing $16 S$ rRNA genes in metagenomic

717 data. Bioinformatics. 2015;31:i35-43.

718 41. Benítez-Páez A, Portune KJ, Sanz Y. Species-level resolution of 16S rRNA gene

719 amplicons sequenced through the MinION ${ }^{\top M}$ portable nanopore sequencer.

$720 \quad$ Gigascience. 2016;5.

721

42. Martijn J, Lind AE, Schön ME, Spiertz I, Juzokaite L, Bunikis I, et al. Confident phylogenetic identification of uncultured prokaryotes through long read amplicon sequencing of the 16S-ITS-23S rRNA operon. Environ Microbiol. John Wiley \& Sons, Ltd; 2019;21:2485-98.

43. Okazaki Y, Fujinaga S, Salcher MM, Callieri C, Tanaka A, Kohzu A, et al.

Microdiversity and phylogeographic diversification of bacterioplankton in pelagic freshwater systems revealed through long-read amplicon sequencing. bioRxiv. 2020;2020.06.03.133140.

44. Zwirglmaier K, Heywood JL, Chamberlain K, Woodward EMS, Zubkov M V.,

730 Scanlan DJ. Basin-scale distribution patterns of picocyanobacterial lineages in the

731 Atlantic Ocean. Environ Microbiol. 2007;9:1278-90.

45. Zwirglmaier K, Jardillier L, Ostrowski M, Mazard S, Garczarek L, Vaulot D, et al.

733 Global phylogeography of marine Synechococcus and Prochlorococcus reveals a

734 distinct partitioning of lineages among oceanic biomes. Environ Microbiol. John 
Wiley \& Sons, Ltd; 2008;10:147-61.

46. Giovannoni SJ. SAR11 Bacteria: The Most Abundant Plankton in the Oceans.

47. Ghai R, Mizuno CM, Picazo A, Camacho A, Rodriguez-Valera F. Metagenomics uncovers a new group of low GC and ultra-small marine Actinobacteria. Sci Rep.

$740 \quad$ Nature Publishing Group; 2013;3:2471.

48. López-Pérez M, Haro-Moreno JM, Iranzo J, Rodriguez-Valera F. Genomes of the Actinomarinales order, highly streamlined actinobacteria adapted to the epipelagic oligotrophic ocean. mSystems.

49. van der Walt AJ, van Goethem MW, Ramond J-B, Makhalanyane TP, Reva O, Cowan DA. Assembling metagenomes, one community at a time. BMC Genomics. $2017 ; 18: 521$.

50. Hauser M, Steinegger M, Söding J. MMseqs software suite for fast and deep clustering and searching of large protein sequence sets. Bioinformatics. $2016 ; 32: 1323-30$.

51. Ramos-Barbero MD, Martin-Cuadrado AB, Viver T, Santos F, Martinez-Garcia M, Antón J. Recovering microbial genomes from metagenomes in hypersaline environments: The Good, the Bad and the Ugly. Syst Appl Microbiol. Elsevier GmbH; 2019;42:30-40.

52. Duarte CM, Ngugi DK, Alam I, Pearman J, Kamau A, Eguiluz VM, et al.

755 Sequencing effort dictates gene discovery in marine microbial metagenomes.

756 Environ Microbiol. John Wiley \& Sons, Ltd; 2020;n/a.

757 53. Biller SJ, Berube PM, Dooley K, Williams M, Satinsky BM, Hackl T, et al. Data descriptor: Marine microbial metagenomes sampled across space and time. Sci Data. Nature Publishing Group; 2018;5:180176. 
54. Acinas SG, Sánchez P, Salazar G, Cornejo-Castillo FM, Sebastián M, Logares R, et al. Metabolic Architecture of the Deep Ocean Microbiome. bioRxiv. 2019;635680.

55. Rusch DB, Halpern AL, Sutton G, Heidelberg KB, Williamson S, Yooseph S, et al.

The Sorcerer II Global Ocean Sampling expedition: northwest Atlantic through eastern tropical Pacific. PLoS Biol. Public Library of Science; 2007;5:e77.

56. Béjà O, Suzuki MT, Hadd A, Nguyen LP, Spudich JL, Spudich EN, et al. Bacterial

57. Fuhrman J a, Schwalbach MS, Stingl U. Proteorhodopsins: an array of physiological roles? Nat Rev Microbiol. 2008;6:488-94. and Archaeal lon-Pumping Rhodopsins: Genetic Diversity, Physiology, and Ecology. Microbiol Mol Biol Rev. 2016;80:929-54.

59. Deisseroth K. Optogenetics. Nat Methods. 2011;8:26-9.

60. Govorunova EG, Sineshchekov OA, Li H, Spudich JL. Microbial Rhodopsins: Reviews; 2017;86:845-72.

61. Finkel OM, Béjà O, Belkin S. Global abundance of microbial rhodopsins. ISME J. 2013;7:448-51.

62. Bratanov D, Kovalev K, Machtens J-P, Astashkin R, Chizhov I, Soloviov D, et al.

780 Unique structure and function of viral rhodopsins. Nat Commun. 2019;10:4939.

782 RubyACRs, nonalgal anion channelrhodopsins with highly red-shifted absorption.

783 Proc Natl Acad Sci. 2020;117:22833 LP - 22840.

784 64. Miyanaga A. Structure and function of polyketide biosynthetic enzymes: various 
strategies for production of structurally diverse polyketides. Biosci Biotechnol

Biochem. Taylor \& Francis; 2017;81:2227-36.

65. Nivina A, Yuet KP, Hsu J, Khosla C. Evolution and Diversity of Assembly-Line

Polyketide Synthases. Chem Rev. American Chemical Society; 2019;119:12524-47.

66. Coates RC, Podell S, Korobeynikov A, Lapidus A, Pevzner P, Sherman DH, et al. $2017 ; 542: 237-41$.

68. Horvath P, Barrangou R. CRISPR/Cas, the Immune System of Bacteria and

Archaea. Science (80- ). 2010;327:167 LP - 170.

69. Biswas A, Staals RHJ, Morales SE, Fineran PC, Brown CM. CRISPRDetect: A flexible algorithm to define CRISPR arrays. BMC Genomics. BioMed Central Ltd.; $2016 ; 17: 356$.

800 70. Mizuno CM, Rodriguez-Valera F, Kimes NE, Ghai R. Expanding the Marine

801 Virosphere Using Metagenomics. Rocha EPC, editor. PLoS Genet. Public Library of Science; 2013;9:e1003987.

71. Mizuno CM, Ghai R, Saghaï A, López-García P, Rodriguez-Valeraa F. Genomes

804 of abundant and widespread viruses from the deep ocean. MBio. 2016;7:1-9.

805 72. Rocap G, Larimer FW, Lamerdin J, Malfatti S, Chain P, Ahlgren NA, et al.

806 Genome divergence in two Prochlorococcus ecotypes reflects oceanic niche

807 differentiation. Nature. Nature Publishing Group; 2003;424:1042-7.

808 73. Coleman ML, Sullivan MB, Martiny AC, Steglich C, Barry K, Delong EF, et al.

809 Genomic Islands and the Ecology and Evolution of Prochlorococcus. Science (80- ). 
811 74. Avrani S, Wurtzel O, Sharon I, Sorek R, Lindell D. Genomic island variability

812 facilitates Prochlorococcus-virus coexistence. Nature. 2011;474:604-8.

813 75. Ahlgren NA, Chen Y, Needham DM, Parada AE, Sachdeva R, Trinh V, et al.

814 Genome and epigenome of a novel marine Thaumarchaeota strain suggest viral

815 infection, phosphorothioation DNA modification and multiple restriction systems.

816 Environ Microbiol. 2017;19:2434-52.

817 76. Rodriguez-Valera F, Martin-Cuadrado A-B, López-Pérez M. Flexible genomic

818 islands as drivers of genome evolution. Curr Opin Microbiol. 2016;31:154-60.

819 77. López-Pérez M, Martin-Cuadrado AB, Rodriguez-Valera F. Homologous recombination is involved in the diversity of replacement flexible genomic Islands in 821 aquatic prokaryotes. Front Genet. Frontiers Media SA; 2014;5:1-1. Bacterium Alteromonas. Genome Biol Evol. 2016;8:1556-70.

79. Neuenschwander SM, Ghai R, Pernthaler J, Salcher MM. Microdiversification in genome-streamlined ubiquitous freshwater Actinobacteria. ISME J. Nature Publishing Group; 2018;12:185-98.

80. Kashtan N, Roggensack SE, Rodrigue S, Thompson JW, Biller SJ, Coe A, et al.

828 Single-cell genomics reveals hundreds of coexisting subpopulations in wild

829 Prochlorococcus. Science. 2014;344:416-20.

830 81. Jayaraman J, Jones WT, Harvey D, Hemara LM, McCann HC, Yoon M, et al.

831 Variation at the common polysaccharide antigen locus drives lipopolysaccharide

832 diversity within the Pseudomonas syringae species complex. Environ Microbiol. John

833 Wiley \& Sons, Ltd; 2020;n/a.

834 82. Holt KE, Lassalle F, Wyres KL, Wick R, Mostowy RJ. Diversity and evolution of 

30. Cuadrado A-B. New insights into marine group III Euryarchaeota, from dark to light. ISME J. 2017;1-16.

84. De Coster W, D’Hert S, Schultz DT, Cruts M, Van Broeckhoven C. NanoPack: visualizing and processing long-read sequencing data. Bioinformatics.

85. Rice P, Longden I, Bleasby A. EMBOSS: The European Molecular Biology Open Software Suite. Trends Genet. 2000;16:276-7.

86. Bolger AM, Lohse M, Usadel B. Trimmomatic: a flexible trimmer for Illumina sequence data. Bioinformatics. Oxford University Press; 2014;30:2114-20. single-cell and metagenomic sequencing data with highly uneven depth. Bioinformatics. 2012;28:1420-8.

850 88. Bankevich A, Nurk S, Antipov D, Gurevich AA, Dvorkin M, Kulikov AS, et al. SPAdes: A New Genome Assembly Algorithm and Its Applications to Single-Cell Sequencing. J Comput Biol. 2012;19:455-77.

854 metaFlye: scalable long-read metagenome assembly using repeat graphs. Nat 855 Methods. 2020;17:1103-10.

856 90. Nurk S, Walenz BP, Rhie A, Vollger MR, Logsdon GA, Grothe R, et al. HiCanu:

857 Accurate assembly of segmental duplications, satellites, and allelic variants from 858 high-fidelity long reads. Genome Res. Cold Spring Harbor Laboratory Press;

$8592020 ; 30: 1291-305$. 
860 91. Hyatt D, Chen G-L, Locascio PF, Land ML, Larimer FW, Hauser LJ. Prodigal:

861 prokaryotic gene recognition and translation initiation site identification. BMC

862 Bioinformatics. 2010;11:119.

863 92. Lowe TM, Eddy SR. TRNAscan-SE: A program for improved detection of transfer

864 RNA genes in genomic sequence. Nucleic Acids Res. 1996;25:955-64.

865 93. Buchfink B, Xie C, Huson DH. Fast and sensitive protein alignment using

866 DIAMOND. Nat Methods. 2015;12:59-60.

867 94. Tatusov RL, Natale DA, Garkavtsev I V, Tatusova TA, Shankavaram UT, Rao BS,

868 et al. The COG database: new developments in phylogenetic classification of

869 proteins from complete genomes. Nucleic Acids Res. 2001;29:22-8.

870 95. Haft DH, Loftus BJ, Richardson DL, Yang F, Eisen JA, Paulsen IT, et al.

871 TIGRFAMs: a protein family resource for the functional identification of proteins.

872 Nucleic Acids Res. 2001;29:41-3.

873 96. Eddy SR. Accelerated profile HMM searches. Pearson WR, editor. PLoS Comput

874 Biol. Washington University School of Medicine, Washington University in St. Louis;

$875 \quad 2011 ; 7: e 1002195$.

876 97. Edgar RC. Search and clustering orders of magnitude faster than BLAST.

877 Bioinformatics. 2010;26:2460-1.

878 98. Quast C, Pruesse E, Yilmaz P, Gerken J, Schweer T, Yarza P, et al. The SILVA 879 ribosomal RNA gene database project: Improved data processing and web-based 880 tools. Nucleic Acids Res. Oxford University Press; 2013;41:D590-6.

881 99. Pruesse E, Peplies J, Glöckner FO. SINA: Accurate high-throughput multiple

882 sequence alignment of ribosomal RNA genes. Bioinformatics. 2012;28:1823-9.

883 100. Nguyen LT, Schmidt HA, Von Haeseler A, Minh BQ. IQ-TREE: A fast and

884 effective stochastic algorithm for estimating maximum-likelihood phylogenies. Mol 
Biol Evol. Narnia; 2015;32:268-74.

886 101. Lê S, Josse J, Husson F. FactoMineR: An R Package for Multivariate Analysis.

887 J Stat Softw. 2008;25:1-18.

888 102. Altschul SF, Madden TL, Schäffer AA, Zhang J, Zhang Z, Miller W, et al.

889 Gapped BLAST and PSI-BLAST: A new generation of protein database search

890 programs. Nucleic Acids Res. 1997;25:3389-402.

891 103. Parks DH, Imelfort M, Skennerton CT, Hugenholtz P, Tyson GW. CheckM: assessing the quality of microbial genomes recovered from isolates, single cells, and metagenomes. Genome Res. 2015;25:1043-55.

104. Richter M, Rossello-Mora R. Shifting the genomic gold standard for the prokaryotic species definition. Proc Natl Acad Sci. National Academy of Sciences; 2009;106:19126-31.

105. Huang Y, Niu B, Gao Y, Fu L, Li W. CD-HIT Suite: A web server for clustering and comparing biological sequences. Bioinformatics. Oxford University Press; 2010;26:680-2.

900 106. Yin Y, Mao X, Yang J, Chen X, Mao F, Xu Y. DbCAN: A web resource for automated carbohydrate-active enzyme annotation. Nucleic Acids Res. 2012;40. 107. Lombard V, Golaconda Ramulu H, Drula E, Coutinho PM, Henrissat B. The carbohydrate-active enzymes database (CAZy) in 2013. Nucleic Acids Res. 2014;42. 108. Blin K, Shaw S, Steinke K, Villebro R, Ziemert N, Lee SY, et al. antiSMASH 5.0: updates to the secondary metabolite genome mining pipeline. Nucleic Acids Res. 2019;47:W81-7. 109. Couvin D, Bernheim A, Toffano-Nioche C, Touchon M, Michalik J, Néron B, et al. CRISPRCasFinder, an update of CRISRFinder, includes a portable version, 909 enhanced performance and integrates search for Cas proteins. Nucleic Acids Res. 
$910 \quad$ 2018;46:W246-51.

911 110. Coutinho FH, Edwards RA, Rodríguez-Valera F. Charting the diversity of

912 uncultured viruses of Archaea and Bacteria. BMC Biol. 2019;17:109.

913

914

915 SUPPLEMENTARY MATERIAL

916 Additional File 1: Figure S1. Summary of the base-call accuracy, converted from the

917 phred score, of the Nanopore raw reads. Upper and right histograms represent length

918 and base-call accuracy distributions, respectively. Figure S2. Histogram

919 representation of the GC content of the three sequencing methods: Illumina (black),

920 Nanopore (red) and PacBio (blue). The resulting GC content after read correction of

921 LR samples are also represented as dashed lines. Table S1. Relative abundance of

922 16S rRNA reads, based on the SILVA database. Table S2. Summary statistics of the

923 assembly of PacBio raw reads using three assemblers (metaSPAdes, metaFlye and

924 CanuHiFi) and applying different subsets of reads. Table S3. Genome parameters of

925 MAGs recovered in this study with ANI $>99.5 \%$ to MAGs retrieved from the same sampling site in the Mediterranean Sea. 